\title{
The Paschalidis Tri-Anthropo-Type Model in Education: Three Learning Profiles, Three Teaching Styles
}

\author{
George Pashalidis \\ Delphon 23C, Pilaia, Thessaloniki, Greece
}

\begin{abstract}
This project demonstrates the Paschalidis TriAnthropo-Type Model in Education as a unique, innovative approach to teaching, learning and parenting/caring, which not only can promote successful teaching and learning but also -and more importantly- guarantee a happy, creative and successful existence for everyone involved in any collaborative community of learning, namely students, teachers and parents/guardians. It also demonstrates that the implementation of the Model cannot be seen just as an "option extra", but rather as a fundamental springboard for successful teaching, learning and parenting/caring, based on self-awareness and heteroawareness of every member of a student's learning environment. The Model identifies only three distinct human personality Types, having specific neurobiological origins, and therefore only three learning profiles, three teaching and three parenting styles. It allows teachers to know their own and each of their students' personality Types and to use this knowledge to improve their teaching significantly for the benefit of each one of their students, no matter their number in the class. This knowledge affords them also to identify easily each student's strengths and weaknesses, skills and abilities. The implementation of the Model enables learners of any age and level to improve their learning, social skills, personality development and psychological well-being. This project focuses particularly on the teaching and learning of Early Childhood, Primary and Secondary Education children in the classroom, which is regarded as a collaborative learning community of students and teachers.
\end{abstract}

\section{Introduction}

Various models have been used to categorize the learning profiles, but only few have taken into account the role the teachers' and students' personalities play in the teaching - learning process [1]. Previous studies and research in the field have investigated factors such as student diversity, learning profiles, brain function and multiple intelligences as dynamics propelling the shift to differentiation in teaching and learning [2]. Contemporary educational projects have focused on brain research and neuroscience to encompass differentiated instruction, so that all learners could be reached [3]. According to these frameworks, educational strategies nowadays are based on the fact that individual differences in learning profiles derive from the fact that learners have diverse minds [4]. The Paschalidis Tri-Anthropo-Type Model in Education brings the balance between oversimplified approaches suggesting that all students are the same and differentiated approaches according to which every student is completely different [5]. According to G. D. Paschalidis "There is only one barrier to learning, and that is the lack of knowledge of the Three Human Personality Types" [6].

In this paper, therefore, the Paschalidis TriAnthropo-Type Model will be reviewed first, followed by the rationale and the aims of the project. A brief description of what actually happened during the project follows, as well as an estimation of whether and to what extend the aims of the project were fulfilled.

\section{The Paschalidis Tri-Anthropo - Type Model and its application in Education}

Various personality-Type models have been developed from ancient times to the present. Personality typologies reveal and increase the knowledge and understanding of individual differences as well as contribute to self-awareness and heteroawareness [7].

The Paschalidis Tri-Anthropo-Type Model is a novel discovery, which unravels the function of the human brain and reveals how personality and learning are interrelated. According to the Model, every human being belongs to only one of three distinct personality Types, namely Type A, Type B or Type C [7]. Each Type is characterised by certain biological and personality traits. Every person inherits genetically their Type (A, B or C) from only one of their biological parents. People who inherit their Type from their father are naturally inclined rather to action than to management/administration, whereas people who inherit their Type from their mother are naturally inclined rather to management than to action [8].

The differences among the three personality Types derive from the different plasticity and connectivity of three specific regions and cortical areas of the limbic system, namely the entorhinal cortex, the amygdalecingulate gyrus and the hippocampus. 
When under stress, the entorhinal cortex over functions in Type A individuals, the amygdalecingulate gyrus over functions in Type B individuals, whereas the hippocampus over functions in Type $\mathrm{C}$ individuals [8].

G. D. Paschalidis writes about Type A individuals: "According to my research, Type A individuals function with the brain area called "endorhinal cortex". They are visual and process information instantly, which leads to their acting immediately. The endorhinal cortex activates the serotonin receptors, resulting in the release of the amount of serotonin needed by the body to perform actions fast. As a result Type A individuals are practical, spontaneous, swift and highly productive. Their haste leads them quickly to success and they are optimists, impulsive, innovative and sociable" [9].

However, according to G. D. Paschalidis, when they are under pressure, they become tense. Any increase of their tension results in increase of the serotonin levels in their body. As a result, they become impatient, show zero tolerance to pressure, make abrupt, clumsy and uncoordinated movements. Their tension forces them to quit easily and keeps them away from success. Under conditions of extreme pressure Type A individuals experience a specific kind of stress, defined by G. D. Paschalidis as "stress of panic of action". This condition either leads them to quick, careless, nonproductive action or to a reaction of giving up and complete inactivity [8]

As far as Type B individuals are concerned, G. D. Paschalidis writes: According to my discovery, Type B individuals function with the brain area called "amygdale". They are auditory and process information cautiously, which leads to their taking delayed action. The amygdale activates the dopamine receptors, resulting in the release of the amount of dopamine needed by the body to perform actions cautiously and lead them to high performance. As a result Type B individuals are theoretical, cautious, hesitant, and slowly productive. Their hesitation and paying attention to detail delays their reaching success and they are doubtful, conservative, reserved and observant [9].

However, according to G. D. Paschalidis, when they are under pressure, they become more observant. Any increase of their observing results in increase of the dopamine levels in their body. As a result, they worry and become watchful, cautious to avoid making mistakes, introverted, indecisive and anxious about the future. Their constant worry and thinking constitutes an obstacle to their achieving their goals. Under conditions of extreme pressure, Type $\mathrm{B}$ individuals experience a specific kind of stress, defined by G. D. Paschalidis as "stress of anxiety". This condition leads them to unproductive repeated cycles of thinking, planning and checking, so that they become unable to make neither any decision or take any action [8].

As far as Type C individuals are concerned, G. D. Paschalidis writes: According to my discovery, C Type individuals function with the brain area called "hippocampus". They are kinesthetic and process information persistently and obsessively in order to reach their goals; they are extreme. The hippocampus activates successively the serotonin and dopamine receptors, resulting in the successive release of serotonin and dopamine needed by the body to function. When the circulation of these hormones is kept at normal levels, they are led to act quickly and efficiently. When they are not under stress, they have high tolerance to pressure, an ability to overcome obstacles, act quickly and effectively and defy danger. They are hard-working perfectionists [9].

When C Type individuals are under pressure, excess amount of both serotonin and dopamine is successively released in their bodies. As a result, a great amount of adrenaline is also released, which makes them lose control of their behavior. Under conditions of extreme pressure, Type $\mathrm{C}$ individuals experience a specific kind of stress, defined by G. D. Paschalidis as "stress of persistence of performance/stubbornness of execution" leads them to persist obsessively in what they consider as the only "right" way of acting, which happens to be the most difficult one, unable to see alternatives. As a result they cannot proceed towards fulfillment of their goals [8].

Apart from the above-mentioned differences in brain structure and function, the three human personality Types are also classified according to other factors, such as: skeletal structure, enterotype, absorption of bio metals and vitamins, predisposition to and manifestation of certain illnesses/disorders, personality traits, behavior and motivation, emotional response to stress as well as cognitive and learning abilities [8]. (It is beyond the scope of this paper to elaborate further on every individual classifying element. Such elaboration can be found in Paschalidis' books).

The Paschalidis Tri-Anthropo-Type Model, therefore, links human brain to personality, behavior, ways of perception, information processing, responding to stimuli, acting and administration as well as emotional and biological functions. Apart from identifying specific differences among students and their learning profiles, teachers and their teaching styles as well as parents/guardians and their parenting/caring styles, it also reveals the neurobiological origins of these differences, thus offering an explanation for the students', teachers', parents' and guardians' mind diversity [6].

Although the application of the Paschalidis TriAnthropo-Type Model in Education involves every member of the learning community of students, teachers and parents/guardians, this paper, due to its limited scope, focuses particularly on students and teachers.

The Paschalidis Tri-Anthropo-Type Model was considered as a unique tool, which could be used in education, in order to help educators reach a better understanding of the differences among learning profiles as well as teaching styles. It could also reveal how these differences affect learning and how Typespecific differentiated instruction can remodel certain neural circuits involved in the teaching-learning processes, via brain structural plasticity. The structure of the brain can be changed, thus influencing the development of life-long learning abilities. 


\subsection{The Rationale of the Project}

The project addressed the issue of teaching diverse minds. Research has shown that a teacher's difficulty to reach all their students in a classroom leads to some students' underperforming, consequent disappointment and unhappiness [10]. Despite the fact that there are several models of differentiated teaching, which suggest taking into account every single student's specific strengths, weaknesses and needs it has been proved practically impossible to individualize instruction for 20-30 students in a typical class.

We started with the assumption, based on experience and previous research, that approaching every student in ways suitable to their personality and learning profile improves their learning, selfconfidence, social skills and overall happiness. Recent research has focused on the role of the personality and individual variability during the learning process [11].

\subsection{The Aims of the Project}

The main aims of the project were:

- To design a differentiated instructional strategy based on the Paschalidis Tri-Anthropo-Type Model and develop appropriate material (i.e lesson plans, team-work guidelines etc) in order to implement this strategy across the curriculum.

- To use qualitative and quantitative observations, questionnaires, grading scales for school performance and interview data to evaluate the implementation of the Paschalidis Tri-AnthropoType Model in Education.

- To disseminate the findings of the project, so that there is an impact on educational research, policies and practices as well as on teacher training at both national and international level.

\section{Methodology}

The participants of our study were forty (40) teachers and three hundred and seventy children (370) with no diagnosed disorder, in eighteen (18) "target classes" in twelve (12) schools, seven (7) of them of primary and five (5) of secondary education, in the prefectures of Western Greece and Western Macedonia, in Greece.

Nineteen (19) of these children were examined and assessed at the "Centre of Counselling and Psychotherapy for the Child and the Family", where the child psychiatrist suggested that it would be beneficial for them to be approached and instructed according to the Paschalidis Tri-Anthropo-Type Model, and collaborated with their teachers in order for them to apply the Model to their teaching. Following this, the differentiated instruction plan was applied in the whole classes these children attended. School heads, parents/guardians and students involved gave their informed consent.

The control group (not subjected to the Paschalidis Tri-Anthropo-Type Model in Education instructional strategy) consisted of twelve (12) randomly selected classes, one in each of the twelve (12) schools.

\subsection{Preparation}

We introduced the Paschalidis Tri-Anthropo-Type Model to the teachers through differentiated, Typebased instruction, providing them with lesson planning guidelines, worksheets and other tutoring on teamwork, collaborative learning etc. Students were assessed at the beginning of the school year 2011-2012, with a second assessment taking place at the end of the same school year. The following were used:

- The Paschalidis Tri-Anthropo-Type Model Questionnaire for Adults

- The Paschalidis Tri-Anthropo-Type Model Questionnaire for Children and Young People

- The Paschalidis' Set of Fairy Tales for Children [12]

- The Achenbach Child Behaviour Checklist (CBCL)

- The Paschalidis Tri-Anthropo-Type Learning Profile Classification

- The Wechsler Intelligence Scale for Children (WISC)

- The Differential Ability Scales-II (DAS-II)

- The Student Progress Assessment Scale

\subsection{Implementation}

The implementation of the Paschalidis TriAnthropo-Type Model in the classroom consists of the following main steps:

First Step: Teachers are introduced to the Paschalidis Tri-Anthropo-Type Model-in Education. They identify their own personality Type, recognize and learn about their Type specific strengths and weaknesses. Colleagues (in the same and in different schools) identify each other's Type, thus facilitating cooperation among them. Self- knowledge in the form of recognition of one's strengths and weaknesses is the first step towards making the maximum of the former and eliminating the latter.

Second Step: Teachers introduce the Model to their students and help them identify their personality Types. As a result, every student in a classroom knows their Type and learns about it. Such self - knowledge leads to the recognition of both their strengths and weaknesses and can be followed by their conscious effort to maximize the former and minimize the latter, in order to achieve better performance. Students also learn the personality Types of their teachers and classmates.

Third Step: Teachers introduce the Model to the parents/guardians of their students. As a result, every parent/guardian recognises their own personality Type and learns about it, as well as the Types of their children and their children's teachers. Apart from increasing their self-knowledge, they find themselves in a position to know their children better, recognize their strengths and weaknesses, explain why they are the way they are, so that they can guide them efficiently and effectively.

Fourth Step: Teachers use in their teaching the Differentiated Instruction \& Collaborative Learning Guidelines for Teachers, the Paschalidis Tri-AnthropoType Model Lesson Planning Guide and certain Three- 
Type Worksheets for different school subjects. This educational material has been developed by George Paschalidis and is based on the Paschalidis TriAnthropo- Type Model, according to which there are only three student personality Types and subsequent learning profiles.

The Paschalidis' Differentiated Instruction and Collaborative Learning Guidelines for Teachers consist of: i) detailed description of the teaching and behavioural style of each one of the three distinct teacher personality Types, ii) detailed description of the learning profile and behavioural style of each one the three distinct student personality Types, iii) specific advice on what each teacher personality Type needs to work on in order to make their teaching more effective and their students more receptive, more collaborative and happier human beings, iv) guidelines on group forming and teamwork, in order for both individual and collaborative learning to be achieved for every student in a class. According to G. D. Paschalidis the working groups in a classroom should be formed by one specific criterion: the students' personality Types. The "ideal group" should consist of one Type A student, two Type B students and three Type C students, with the Type A students moving among groups (see 3.6.), v) analyses of the behavioural phenotype, role in the group, motivation, materials and ways in which a teacher can help every student personality Type in collaborative learning groupings.

\subsection{Phase 3: Analysis and Dissemination}

The students were assessed again at the end of the school year 2011-2012. The analyses of the records during this research were introduced to the public and the scientific community through presentations and publications. The results of the analyses are briefly described in the following section.

\section{Results}

The personality Type of each student as well as their learning Profile was found unchanged at the time of the second assessment. A significant correlation $(p<0,05)$ of the three personality Types $(A, B$ and $C)$ with the three learning Profiles (A, B and C) was confirmed. This finding suggests that personality Types and learning Profiles remain unchanged across time.

All students, regardless of their personality Type, showed a significant improvement of the scores in the Student Progress Assessment Scale after the implementation of the Paschalidis Tri-Anthropo-Type Model-in Education.

During the project we developed detailed tables concerning : I) teachers and II) students listing i) Typespecific teachers' personality traits, ii) Type- specific advice for teachers, iii) Type- specific students' personality traits, iv) Type-specific students' cognitive characteristics, v) Type- specific learning characteristics vi) Type- specific students' behaviour in the classroom, vii) Type-specific students' ways of studying and viii) Type-specific students' psychological characteristics. These tables demonstrate how teachers and students act, work, respond to stress, etc.

Elements of the project's findings concerning the above are cited below.

\subsection{Type- specific teachers' personality traits}

Type A teachers, for example, tend to be laconic and avoid elaboration; they analyze only the key points of what they teach and consider many things as obvious. Type B tend to be analytical and meticulous; they explain very carefully and in detail. Type $C$ tend to overload students with information and impose it on them.

Type A teachers don't always follow the given curriculum; they teach what they consider as useful for their students. Type B teachers always follow the given curriculum; they teach what has to be taught, thus avoiding getting in trouble. Type $\mathrm{C}$ teachers follow the curriculum and bring much more into it.

Type A teachers evaluate their students' performance based rather on the overall picture than on specific tasks, whereas Type B base evaluate it based rather on specific tasks than on the overall picture, and Type $\mathrm{C}$ teachers based on both the overall picture and specific tasks.

Type A teachers encourage their students' selfmotivation and offer their help only at difficult points, whereas Type B teachers do not encourage their students' self-motivation, because they are concerned about possible consequences, and Type $\mathrm{C}$ teachers not only encourage their students' self-motivation but also guide them towards their own ways of working and are too demanding of them.

Type A teachers are impatient and edgy, Type B are patient and nervous, whereas Type $\mathrm{C}$ teachers are persistent and obsessive. Type A teachers are practical, whereas Type B are theoretical and Type $\mathrm{C}$ are both practical and theoretical.

Type A teachers improvise, teach freely and creatively and convey their attitude to their students, whereas Type B teach according to plan, are careful, worry about details and convey their worry to their students, and Type $\mathrm{C}$ teachers teach according to plan, used controlled improvisation, stick to detail and exhaust their students.

Type A teachers reward generously, whereas Type $B$ reward cautiously and Type $C$ very selectively. Type A teachers are spontaneous, whereas Type B are reserved and Type $\mathrm{C}$ are stubborn.

\subsection{Type- specific advice for teachers}

In order for their teaching to be more effective, therefore, teachers first of all need to be aware of the specific traits of the personality Type they belong to.

This knowledge will show Type A teachers, for example, that they need to smile more often and be more patient, so that their movements become calmer and more relaxed. It will also show them that they need to control their tension, be less abrupt, more analytical and explanatory. 
Type B teachers will realize, amongst others that they need to dare be innovative and creative, less analytical, more persistent, more confident, trust their students and offer them encouragement.

Type $\mathrm{C}$ teachers will become aware of the fact that they need to be more flexible, less strict and demanding, give their students more space to express themselves and avoid punishing them.

\subsection{Type - specific students' personality traits}

Type A students are self -confident, sensitive and efficient, sociable, communicative, cooperative, decisive, restless, irritable, act spontaneously, take action but can easily give up, their movements are clumsy and ungainly.

Type B students are sociable, patient, polite, reserved, cautious, conformist, conservative, introvert, anxious, insecure, their spontaneity is hindered by their relentless thinking, and their movements are cautious and restrained.

Type C students self-confident and single-minded, tough and efficient, hard working and committed sociable, daring, competitive, radical, demanding, meticulous, irritable, tenacious, their spontaneity is hindered by their persistence.

\subsection{Type - specific students' cognitive characteristics}

As far as information processing is concerned, Type A students are visual, practical and quick, whereas Type B are auditory, analytical, slow, processing information successively, and Type C students are kinaesthetic and relatively slow, due to their paying excessive attention to detail.

As far as tolerance to pressure is concerned, Type A students have zero tolerance to pressure, whereas Type B students have medium tolerance to pressure and tend to find themselves lost in their thoughts, and Type C students have high tolerance to pressure and persevere in order to achieve their goals.

In conceptualising an issue Type A students are quick, they immediately see the whole picture, whereas Type B students are slow, tend to analyse and process all the details, and Type $\mathrm{C}$ students begin to see the whole picture, but focus on any problematic details, get stuck in attempting to clarify them and lose it, as a result.

Type A students are motivated by freedom to express themselves and to act, recognition and rewards, whereas Type B students by encouragement, awards and a sense of security, and Type C students are motivated by acceptance and honorary awards.

\subsection{Type - specific students' learning characteristics}

As far as decision making is concerned A Type students make decisions quickly and change their mind easily, whereas Type B students don't make decisions quickly, and Type C students make decisions quickly and don't change their mind.
When working Type A students choose the easy way, whereas Type B contemplate many alternative ways, and Type $\mathrm{C}$ students choose the most challenging way.

In verbal reasoning Type A students are brief and concise, whereas Type B students are analytical and detailed, and Type $\mathrm{C}$ students are eloquent and their reasoning is based on arguments.

\subsection{Type- specific students' behaviour in the classroom}

In group work Type A students generate ideas, whereas Type B manage the ideas offered, and Type C students put them into practice.

During a discussion Type A students are impatient, want to speak first and say what they have to say immediately and in a straightforward manner, whereas Type B students wait for their turn to speak, are wordy and descriptive, and Type $\mathrm{C}$ students are impatient and use body language and dramatic gestures, in order to be better understood.

During the lesson when the teacher asks a question, Type A students raise their hand to answer it, before the teacher has finished phrasing it, whereas Type B students raise their hand only after they have heard the question and made sure that they know the correct answer, and Type $\mathrm{C}$ students raise their hand only after they have heard it and, if they know the answer, they make a fuss and insist to speak.

\subsection{Type- specific students' ways of studying}

Type A students study for a short time, at the last minute and take regular breaks. They learn the main points of a lesson and can present it briefly in their own words. They do the absolutely necessary work, so that they always have free time.

Type B students study for a long time, to ensure they have understood the lesson. They learn a lesson by heart and don't take breaks, because they are too stressed out. Their studying is restricted to the text books, which they repeatedly work on, since they don't want to be caught unprepared.

Type C students study systematically, force themselves to learn what they want to learn and don't take breaks. They learn a lesson thoroughly, but get carried away by their existing knowledge and give their own interpretations. Their studying is not restricted to the textbooks, but is extended, to ensure they are always prepared and ready. They don't have any free time.

\subsection{Type-specific students' psychological characteristics}

Type A students are restless, lose patience, panic, lose control and explode easily and tend to run away.

Type B students are anxious, insecure, too preoccupied, get easily upset, tend to moan, worry and get confused.

Type C students are obsessive, feel rejected, persevere, have a strong sense of justice and an equally 
strong desire for revenge, get stubborn and don't give up easily.

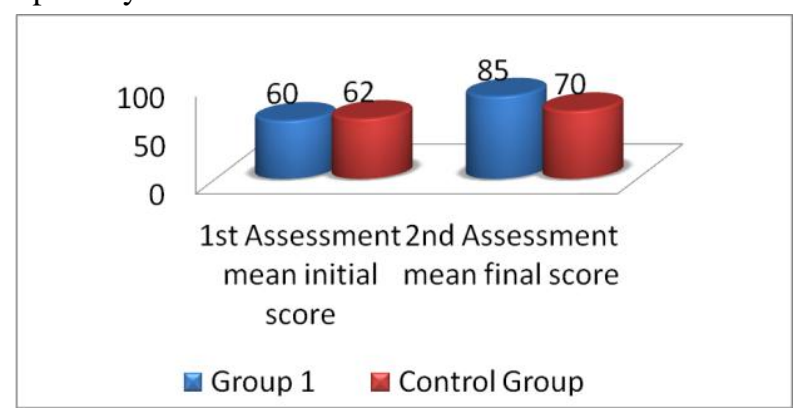

Figure 1. Progress after the implementation of Paschalidis Tri-Anthropo-Type Model

We found that the implementation of the Paschalidis Tri-Anthropo- Type Model in Education improved students' performance, enhanced their learning and increased their grades significantly, in comparison to the Control Group. Furthermore, the data collected from observations of the students and from the interviews with them and their teachers showed that students subjected to the Paschalidis Trianthropo- Type Model had developed self-awareness and hetero-awareness (a better understanding of their classmates, peers, teachers, family members and others) in ways that had helped their personality development, social skills and overall happiness. Secondary education students, in particular, obtained a clearer idea of which profession would be more suitable to their personality Type. They also felt that their acquired knowledge would help them to fulfill the requirements of any profession they would find themselves in the future.

\subsection{Dissemination and Impact}

The outcomes of the project can be summarized as follows:

a) There is evidence of the value of the Paschalidis Tri-Anthropo-Type Model in Education approach for promoting every student's learning, performance and overall happiness.

b) Teachers can use specific strategies to help their students reach their full potential.

c) Findings will be submitted for publication in educational research journals and will be presented in national and international conferences, so that they are shared by researchers and reach stakeholders and policy makers.

d) Findings will also be presented in teacher professional development courses.

e) A book for teachers will be published drawing on the work and the findings of the project.

f) Further research will be conducted focusing on parents/guardians.

\section{Conclusion}

The Paschalidis Tri-Anthropo-Type Model in Education embraces all students worldwide, as it is based on the existence of only three human personality Types and, consequently, only three learning profiles, as well as three teaching and parenting/caring styles. Its implementation in the classroom leads to the creation of a nurturing class and school environment and to a significant improvement of the teacher-student relationship as well as the relationships among classmates and schoolmates, students and their parents, teachers and their students' parents. The Paschalidis Tri-Anthropo-Type Model in Education constitutes a valuable tool for teachers, as it increases their selfawareness by allowing them to identify the roots of their teaching style and behaviour. In a similar way, they can clearly and quickly identify their students' learning profiles and behavioural patterns. This information enhances their instruction strengths, leads to elimination of their weaknesses and enables them to increase the effectiveness of their teaching by determining the optimal teaching strategy for every single student in their class. The teaching - learning process becomes easier and more effective.

Regardless of their age and the grade of education they are in (preschool, primary, secondary), as well as the environment they are taught in, every student belongs to only one of the three personality Types. They all have equal opportunities to fulfil their true potential, on condition that the teaching strategies used are designed taking into consideration their personality Types, as defined, analysed and interpreted by the Paschalidis Tri-Anthropo-Type Model.

It is crucial for teachers to realise that i) their teaching style has been influenced by their personality Type and ii) in-depth knowledge of their personality Type can liberate them from their Types' constraints, iii) their students' learning profiles have been influenced by their personality Type, iv) in-depth knowledge of the three personality Types can give them a detailed and complete picture of their class as a whole and of every single student in a class as a unique human being.

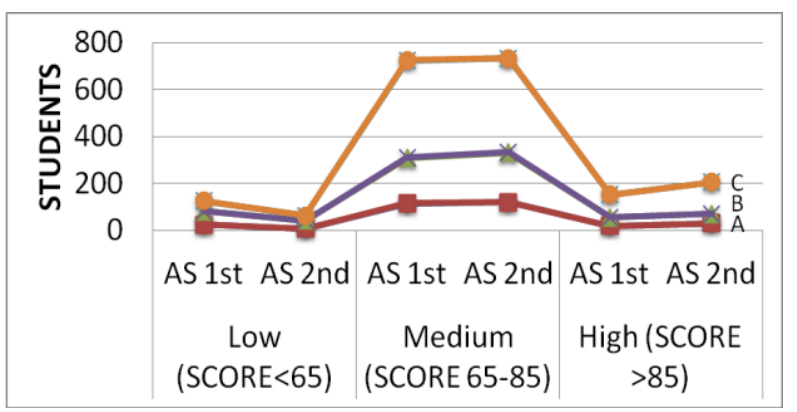

Figure 2. Comparison of students' performance with the control group

It is also of fundamental value for every student to be introduced to the Paschalidis Tri-Anthropo-Type Model in Education, so that i) by developing their self-awareness, unlock their abilities, give prominence to them and reach their true potential [13] ii) by knowing who the significant others in their lives really are (hetero-knowledge) can demystify aspects of their behaviour [14].

The Paschalidis Tri-Anthropo-Type Model in Education develops a positive and fertile educative environment, based on the understanding of self and 
"other", on acceptance, collaboration, praise, discretion and positive reinforcement.

The Paschalidis Tri-Anthropo-Type Model in Education is a great scientific discovery of our age, a multi-dimensional tool applicable to the fields of Education, Health, Psychology, Psychiatry and many more, enabling children, young people and adults to reach their full potential and lead happier lives.

\section{References}

[1] Threeton, M. D. and Walter, R.A. (2009) 'The Relationship between Personality Type and Learning Style: a Study of Automotive Technology Students', Journal of Industrial Teaching Education 46(2), pp. 48-74.

[2] Subban, P. (2006) 'Differentiated Instruction: A Research Basis', International Education Journal 7(7), pp. 935-947.

[3] Tomlinson, C. A. (2003) 'Deciding to Teach Them All', Educational Leadership 61(2), 2003, pp. 6-11.

[4] Tomassini, V. (2011) 'Structural and Functional Bases for Individual Differences in Motor Learning', Human Brain Mapping 32(3), pp.494-508.

[5] Tomlinson, C. A. (2004) 'Differentiation in Diverse Settings', School Administrator 61(7), pp. 28-33.

[6] Stathopoulou A.N. and Pashalidis G.D. (2012) 'The Model of the Three Human Personality Types: a Unique Tool for the Understanding of the Individual Differences in the Teaching-Learning Process', in Proceedings of the 5th International Conference of Education, Research and Innovation, IATED: Madrid, Spain, p.p.2050-2059.

[7] Paschalidis, G. D. (2011) Love Opening Path to Life, Media Vista, Athens.

[8] Paschalidis, G.D. and Stathopoulou A. (2012) 'Genetically Determined Differences of the Three Structures of Limbic Brain System Determine Three Distinctive and Unique Personality Types: a Special Key for Understanding Connection between Brain Function and its Plasticity with Health Prevention', in proceedings of the 18th Stress and Behavior ISBS Conference, ISBS: New Orleans, USA, pp. 22-23.

[9] Paschalidis, G. D. (2012) Character Analysis, Media Vista, Athens (in Greek, under publication in English).

[10] Leaver, B.L. (1998) Teaching the Whole Class, Kendall Hunt, Dubuque, IA.

[11] Busato, V.V. (1998) 'The Relation between Learning Styles, the Big Five Personality Traits and Achievement Motivation in Higher Education', Personality \& Individual Differences 26(1), pp. 129-140.

[12] Paschalidis, G. D. (2012) Set of Fairy Tales, Media Vista, Athens (in Greek).

[13] Paschalidis, G. D. (2012) Choosing a Career, Livanis, Athens (in Greek, under publication in English).

[14] Paschalidis, G. D. (2012) Human Relations, Media Vista, Athens (in Greek, under publication in English). 